\title{
DESIGN, SYNTHESIS AND IN VITRO EVALUATION OF ANTIBACTERIAL AND ANTIADHESIVE POTENTIALS OF SOME SUBSTITUTED IMIDAZOLONES DERIVATIVES OF CINNAMALDEHYDE
}

\author{
BY \\ Abdelsattar M. Omar, ${ }^{*}$, b and Mahmoud A. Elfakyc
}

FROM

a Department of Pharmaceutical Chemistry, Faculty of Pharmacy, King Abdulaziz

University, Alsulaymanyah, Jeddah 21589, Saudi Arabia.

${ }^{\mathrm{b}}$ Department of Pharmaceutical Chemistry, Faculty of Pharmacy, Al-Azhar University, Cairo 11884, Egypt.

${ }^{c}$ Department of Natural Products and Alternative Medicine, Faculty of Pharmacy, King Abdulaziz University, Alsulaymanyah, Jeddah 21589, Saudi Arabia.

\section{ABSTRACT}

The cells of microorganisms often stick together and adhere to biotic and inert surfaces to form coats (biofilms) that are impenetrable and often highly resistant to antibiotics, as well as being adaptive to immune responses of the host. The aim of this study is to develop drugs that are able to prevent cell adhesion and/or penetrate biofilm layer and reach the bacteria to render them harmless. trans-Cinnamaldehyde, found in oils and cinnamon barks, and curcumin found in turmeric have been found to hold such pharmacologic properties. The newly developed product hybrids were evaluated for their ability to exert in vitro antibacterial activity as well as their potential as bacterial anti-adhesive agents on several different bacteria. In summary, we have developed several novel compounds that show both antibacterial and anti-adhesive properties. Although the compounds are not as potent as the positive controls, they serve as lead for further structural modification to develop more potent derivatives.

Key words: Synthesis, Antibacterial, Bacterial Anti-adhesive, biofilm, Imidazolone, Cinnamaldehyde

\section{Introduction}

Almost all microorganisms exert survival mechanism through the formation of biofilms, which is an association of the microbial cells to one another on inert or biological surfaces within a self-produced matrix of extracellular polymeric substance(Sekhar et al., 2009). Biofilms protect its inhabitants from predators, biocides, dehydration and other extreme environmental conditions(Dunne, 2002). Adhesion of the bacteria is the primary step taken by the bacteria that leads to pathogenic infections, and that involves resulting in nosocomial and community infections and posing as a significant threat to public health because of their impenetrable and resistance to most antibiotics(Costerton et al., 1999)' (Khan et al., 2014). Several intrinsic mechanisms have been postulated to explain the high resistance nature of biofilms(Nickel and Costerton, 1993). These include limited diffusion, enzymes that cause neutralization, slow growth rate, biofilm phenotype as adaptive mechanisms and heterogeneous functions (Anderl et al., 2000), (Lewis, 2001),(Stewart and Franklin, 2008),(Estela and Alejandro, 2012). Examples of biofilm-implicated infections are those by E. coli, H. 
influenza, S. aureus, S. epidermidis, P. aeruginosa, causing many diseases with deadly effects if kept untreated(Sekhar et al., 2009), (Larson et al., 2003, Jensen et al., 2007), (Novick and Geisinger, 2008, Lyczak et al., 2002), (Otto et al., 1998, Vadyvaloo and Otto, 2005). (Naves et al., 2010). The extracellular matrix of certain pathogens, e.g. S. aureus biofilms is formed by recycled cytoplasmic proteins, which allows the bacteria to adapt to its environment and also promote production of mixed-species(Foulston et al., 2014).

There is an increasing focus to develop antibiotics that can penetrate the biofilm layer or even eradicate the biofilms encasing the bacteria to make the antibiotics reach their target(Liu et al., 2004, Kim et al., 2012). Curcumin (Fig. 1) an active ingredient of turmeric has been shown to be effective in inhibiting the formation of biofilms; making this compound a potential treatment for pathogens(Karaman et al., 2013). transCinnamaldehyde a constituent of cinnamon bark or found in cinnamon oil has also been shown to significantly decrease the production of two virulence factors, A and $\alpha$-toxin in S aureus(Smith-Palmer et al., 2004). Low levels of trans-cinnamaldehyde have also been noted to inhibit the cell-density dependent on the regulation of gene expression in E. coli and biofilm synthesis in Vibrio spp.(Brackman et al., 2008). Additionally, transcinnamaldehyde has been shown to reduce the expression of several virulence genes essential for Uropathogenic Escherichia coli (UPEC) motility, host cell attachment and invasion. trans-Cinnamaldehyde has been recognized by the FDA as a safe molecule for use in food, and sub-chronic and chronic studies show this compound to have no adverse effects(Adams et al., 2004). These findings suggest that both transcinnamaldehyde and curcumin are potential candidates for the inhibition of biofilm production, as well as anti-adhesive agents(Amalaradjou et al., 2011).

Curcumin and trans-Cinnamaldehyde (Fig. 1) contain a common core structural moiety, phenyl-alkyl-one ; which we propose is important and/or essential for their observed antibiotic pharmacologic activities. We decided to take advantage of this core feature to produce novel agents by hybridizing the phenyl-alkyl-one with substituted imidazolone (Fig. 1). The 3-alkylidene-4-imidazolone skeleton was selected as it possesses a greater size and more importantly contains an already preserved electronwithdrawing substructure. The electron-withdrawing groups in the compounds are expected to improve their metabolic stability, as well as control the electrophilic reactivity of the nucleus molecule while maintaining a "drug-like electrophile" core. The newly developed natural product hybrids were evaluated for their ability to exert in vitro antibacterial activity as well as their potential as bacterial anti-adhesive agents.

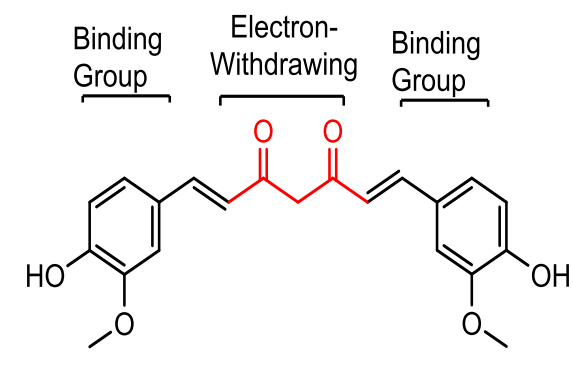

Curcumin

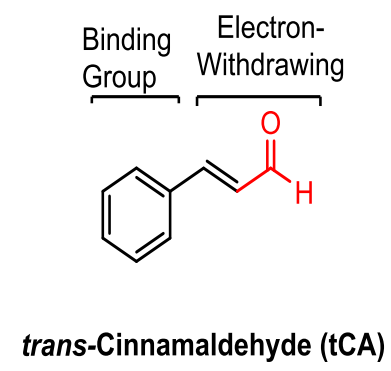

Aldehyde (Non-Drug-Like)

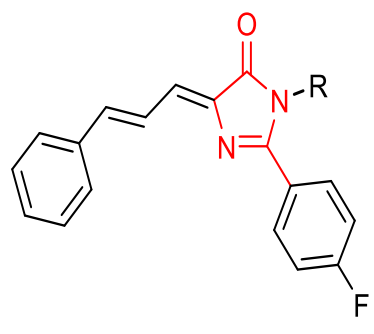

Targetet Imidazolone Derivatives

Figure 1. Design of Curcumin and tCA hybrids.

\section{Results}




\section{Chemistry}

The syntheses of the intermediate and the target compounds are illustrated in scheme 1 . The starting compound 4-fluorobenzoylglycine $\mathbf{1}$ was prepared according to a reported method(El-Araby et al., 2012). Using standard Erlenmeyer methodology (ElAraby et al., 2012, Dakin, 1929), the 4-fluorobenzoylglycine 1 was condensed with cinnamaldehyde 2 to produce the corresponding 2-(4-fluorophenyl)-4-((E)-3phenylallylidene)oxazol-5(4H)-one $\mathbf{3}$. This oxazolone key intermediate $\mathbf{3}$ was subsequently reacted with a variety of aliphatic, aromatic and heterocyclic amines in the presence of dry pyridine to afford the targeted 3-substituted-2-(4-fluorophenyl)-5-((E)3-phenylallylidene)-3,5-dihydro-4H-imidazol-4-ones 4(Lokhandwala and Rai) .
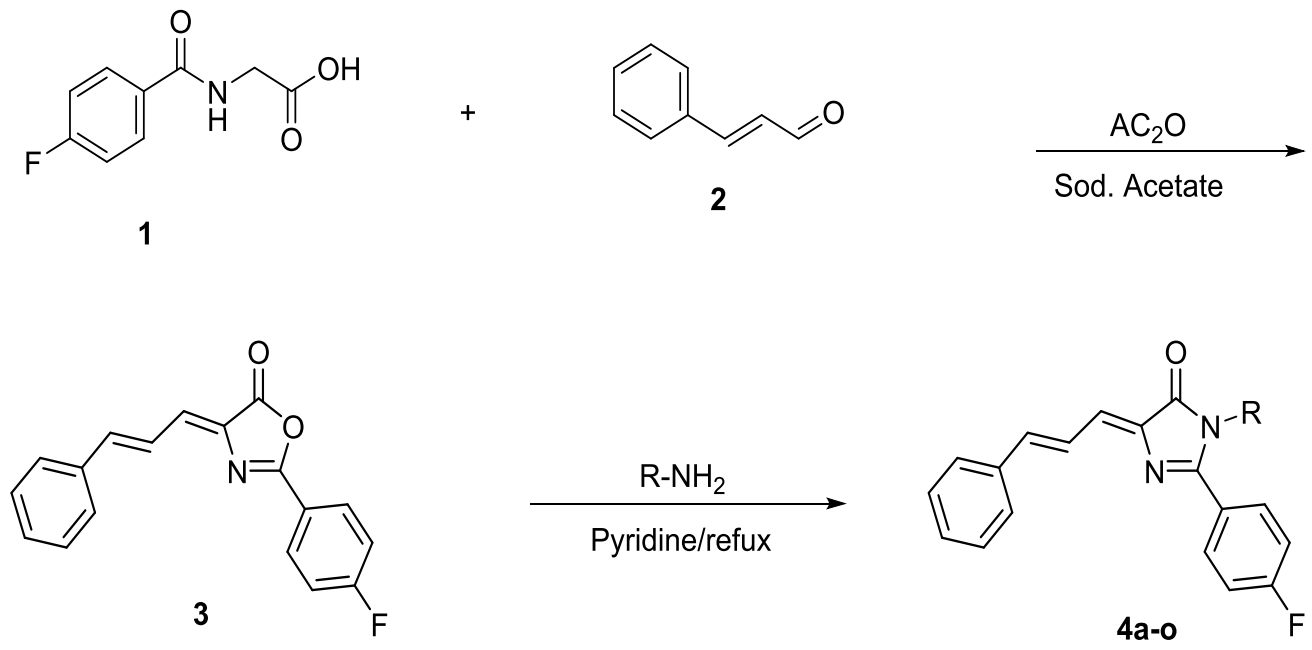

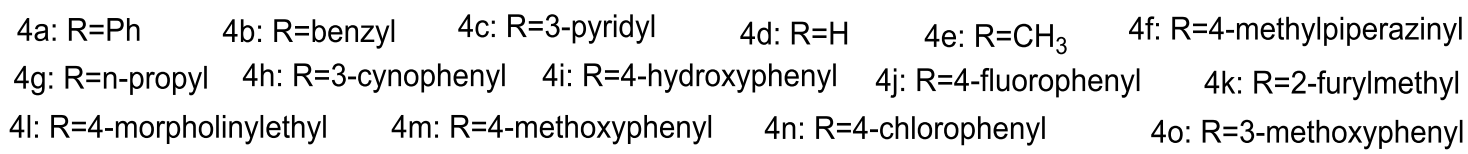

Scheme 1. Synthesis of Imidazolone Derivatives

\section{In vitro Antibacterial Evaluation}

All newly synthesized target compounds 4a-o were tested for their in vitro antibacterial activity against the Gram positive bacteria, Staphylococcus aureus (ATCC 29213), Staphylococcus epidermidis (ATCC 12228) and Bacillus subtilis (ATCC 12228), and the Gram negative bacteria, Escherichia coli (ATCC 35218), Pseudomonas aeruginosa (ATCC 27853), Proteus mirabilis (ATCC 14153) and Klebsiella pneumonia (ATCC 700603) .

As shown in Table 1, the majority of the tested compounds showed antibacterial activity towards most of the microorganisms. Compound $4 \mathbf{d}$ showed the broadest spectrum of activity against several bacteria with MIC values $(62.5 \mu \mathrm{g} / \mathrm{mL}$ against $S$. aureus, $S$. epidermidis, B. subtilis, $P$. aeruginosa and $P$. mirabilis), followed closely by 4a $(62.5 \mu \mathrm{g} / \mathrm{mL}$ against $S$. aureus and against $E$. coli and $125 \mu \mathrm{g} / \mathrm{mL}$ against $P$. mirabilis). Other compounds e.g.4f $(62.5 \mu \mathrm{g} / \mathrm{mL}$ against $\mathrm{S}$. aureus and $125 \mu \mathrm{g} / \mathrm{mL}$ against $P$. mirabilis and E. coli), $4 \mathbf{j}(62.5 \mu \mathrm{g} / \mathrm{mL}$ against $S$. aureus and $125 \mu \mathrm{g} / \mathrm{mL}$ against $P$. mirabilis and $E$. coli) and $4 \mathbf{n}(62.5 \mu \mathrm{g} / \mathrm{mL}$ against $S$. aureus), then $4 \mathbf{i}(125$ 
$\mu \mathrm{g} / \mathrm{mL}$ against $P$. mirabilis $), 4 \mathbf{l}(62.5 \mu \mathrm{g} / \mathrm{mL}$ against $S$. aureus $)$ and $\mathbf{4 m}(125 \mu \mathrm{g} / \mathrm{mL}$ against $P$. mirabilis) also showed significant antibacterial activities, but only against a limited number of the bacteria. Compounds $\mathbf{4 g}(125 \mu \mathrm{g} / \mathrm{mL}$ against $S$. aureus $), \mathbf{4 h}(62.5$ $\mu \mathrm{g} / \mathrm{mL}$ against $S$. aureus $), \mathbf{4 n}(62.5 \mu \mathrm{g} / \mathrm{mL}$ against $S$. aureus $)$ and $\mathbf{3}(125 \mu \mathrm{g} / \mathrm{mL}$ against P. mirabilis) showed the least antibacterial activity for the selected concentration range. These observed MIC results (62.5 or $125 \mu \mathrm{g} / \mathrm{mL}$ ) compare with the MIC values of 0.1 $18 \mu \mathrm{g} / \mathrm{mL}$ observed with ceftriaxone against all the bacteria, and $1.5-12.5 \mu \mathrm{g} / \mathrm{mL}$ with amoxicillin against selected bacteria.

\section{Adherence assay of S. aureus ATCC 29213 to HCT 116 cells}

The assay was carried out as described by Plotkowski et al. (1994) (Plotkowski et al., 1994). Inhibition of adherence of S. aureus ATCC 29213 to HCT 116 ATCC ® CCL-247 ${ }^{\mathrm{TM}}$ human colorectal carcinoma cells was established by incubating constant numbers of HCT 116 cells and bacteria for 2 hours, followed by addition of compounds at concentrations $(6.25,12.5,25$ and $50 \mu \mathrm{M})$ that are harmless to pro- and eukaryotic cells (Table 2). The results showed statistically-significant $(p<0.05)$ inhibition of adherence of $S$. aureus by compounds $4 \mathbf{a}(59.12 \%$ at $25 \mu \mathrm{M}), \mathbf{4 b}(69.57 \%$ at $6.25 \mu \mathrm{M})$, $4 d(51.40 \%$ and $64.41 \%$ at $12.5 \mu \mathrm{M}$ and $50 \mu \mathrm{M}$, respectively), $4 \mathrm{e}(68.22 \%$ at $12.5 \mu \mathrm{M})$, $\mathbf{4 g}(58.88 \%$ and $55.93 \%$ at $12.5 \mu \mathrm{M}$ and $50 \mu \mathrm{M}$, respectively), $\mathbf{4 j}(51.40 \%$ at $12.5 \mu \mathrm{M})$, $4 \mathrm{~m}(55.14 \%$ at $12.5 \mu \mathrm{M})$ and $4 \mathrm{n}(66.36 \%$ and $54.74 \%$ at $12.5 \mu \mathrm{M}$ and $25 \mu \mathrm{M}$, respectively). The results also showed highly statistically-significant $(p<0.01)$ inhibition of the adherence of S. aureus ATCC 29213 to the same human colorectal carcinoma cells by compounds $4 \mathrm{a}(77.57 \%$ and $88.14 \%$ at $12.5 \mu \mathrm{M}$ and $50 \mu \mathrm{M}$, respectively), $4 \mathbf{b}(86.92 \%, 95.62 \%$ and $100.00 \%$ at $12.5 \mu \mathrm{M}, 25 \mu \mathrm{M}$ and $50 \mu \mathrm{M}$, respectively), $\mathbf{4 c}(91.30 \%, 98.13 \%, 98.54 \%$ and $99.15 \%$ at the four concentrations tested), $\mathbf{4 d}(82.48 \%$ at $25 \mu \mathrm{M}), \mathbf{4 e}(86.13 \%$ at $25 \mu \mathrm{M}), \mathbf{4 g}(76.64 \%$ at $25 \mu \mathrm{M}), \mathbf{4 h}$ $(88.32 \%$ at $25 \mu \mathrm{M}), \mathbf{4 i}(64.49 \%$ and $83.94 \%$ at $12.5 \mu \mathrm{M}$ and $25 \mu \mathrm{M}$, respectively), $4 \mathbf{k}$ $(76.64 \%$ at $25 \mu \mathrm{M})$ and $4 \mathrm{n}(80.43 \%$ at $6.25 \mu \mathrm{M})$. However, no inhibitory effect was observed for compounds $\mathbf{4 f}, \mathbf{4 l}, \mathbf{4 o}$ and 3 at any of the concentrations tested (Table 2).

\section{Statistical Analysis}

A one-sample $t$-test was used to evaluate whether the inhibition of adherence of S. aureus ATCC 29213 to HCT 116 ATCC ® CCL-247TM human colorectal carcinoma cells was different from 0\%. The GraphPad Prism program for Windows, Version 6, was used for data processing.

\section{Discussion}

Biofilm formation is used by bacterial and other microbes as a survival mechanism by becoming impenetrable and resistant to antibiotics. Biofilms are also involved in a wide variety of microbial infections. Over the past several years, compounds derived from plants have been shown not only to have antibacterial properties but the ability to inhibit bacterial adhesion and biofilm formation(Palaska et al., 2013). These properties make these compounds display some strong advantages over the use of antibiotics, since, as the adhesion would be prevented, the drug would perform more efficiently at an earlier stage of the disease, and it would not depend on the bacterial multiplication rates and most importantly, would not foster antibiotic resistance development. Therefore, the development of antiadhesives and antibacterial agents would serve as promising solutions for the resistance displayed by the bacteria because of their biofilms. 
In our current study, we have synthesized 3-substituted-2-(4-fluorophenyl)-5((E)-3-phenylallylidene)-3,5-dihydro-4H-imidazol-4-ones derivatives, by hybridizing the cinnamaldehyde/curcumin core structural moiety, phenyl-alkyl-one with substituted imidazolone. We expect these compounds to exhibit antibacterial effect by inhibiting bacterial adhesion and biofilm formation and killing them. When tested against the bacteria, S. aureus, S. epidermidis, B. subtilis, E. coli, P. aeruginosa, P. mirabilis and K. pneumonia, most of the compounds showed antibacterial activity with compound $4 \mathrm{~d}$ showing the broadest spectrum of activity against five of the seven tested bacterial, all with MIC value of $62.5 \mu \mathrm{g} / \mathrm{mL}$ but significantly lower than the observed for ceftriaxone (CTX) and amoxicillin (AMX) (0.14-18) for the same bacteria. Worth mentioning is that 4a showed the highest activity among all the tested compounds against E. coli with MIC of $15.56 \mu \mathrm{g} / \mathrm{mL}$. In addition it inhibited two other bacteria with MIC values of 62.5 and 125 .

It appears that the alkyl or aryl substitution at position 3 of the imidazolone are detrimental for the antibacterial activity while the non-substituted possessed significant antibacterial activity toward the majority of the microorganisms.

Pathogenesis of S. aureus and for that matter, all antibacterial are associated with their adhesive mechanisms and biofilm formation; therefore, the inhibition of the microbes adherence would be a most promising measure of attenuating disease progressions. We measured the average inhibition of adherence of S. aureus. The results showed that most of the compounds presented significant inhibition of adherence, either substituted or non-substituted at position 3 of the imidazolone.

In summary, we have developed several novel compounds that show both antibacterial and anti-adhesive properties. Although the compounds are not as potent as the positive controls, CTX and AMX, they serve as lead for further structural modification to develop more potent derivatives. 
Table 1. Antibacterial activities of the compounds studied

\begin{tabular}{|c|c|c|c|c|c|c|c|c|}
\hline \multirow{3}{*}{ No. } & \multirow{3}{*}{ Code } & \multicolumn{7}{|c|}{ MICs $(\mu \mathrm{g} / \mathrm{mL})$ values } \\
\hline & & \multicolumn{3}{|c|}{ Gram-positive bacteria } & \multicolumn{4}{|c|}{ Gram-negative bacteria } \\
\hline & & $\begin{array}{c}S . \\
\text { aureus } \\
\end{array}$ & $\begin{array}{c}S . \\
\text { epidermidi } \\
\end{array}$ & $\begin{array}{c}B . \\
\text { subtilis } \\
\end{array}$ & $\underline{\text { E. coli }}$ & $\begin{array}{c}K . \\
\text { pneumoni }\end{array}$ & $\begin{array}{c}P . \\
\text { aeruginos }\end{array}$ & $\begin{array}{c}P . \\
\text { mirabilis }\end{array}$ \\
\hline 1 & 3 & $>500$ & $>500$ & $>500$ & $>500$ & $>500$ & $>500$ & 125 \\
\hline 2 & $4 \mathbf{a}$ & 62.5 & $>500$ & $>500$ & 15.56 & $>500$ & $>500$ & 125 \\
\hline 3 & $4 b$ & $>500$ & $>500$ & $>500$ & $>500$ & $>500$ & $>500$ & $>500$ \\
\hline 4 & $4 c$ & $>500$ & $>500$ & $>500$ & $>500$ & $>500$ & $>500$ & $>500$ \\
\hline 5 & $4 d$ & 62.5 & 62.5 & 62.5 & $>500$ & $>500$ & 62.5 & 62.5 \\
\hline 6 & $4 e$ & $>500$ & $>500$ & $>500$ & $>500$ & $>500$ & $>500$ & $>500$ \\
\hline 7 & $4 f$ & 62.5 & $>500$ & $>500$ & 125 & $>500$ & $>500$ & 125 \\
\hline 8 & $4 g$ & 125 & $>500$ & $>500$ & $>500$ & $>500$ & $>500$ & $>500$ \\
\hline 9 & $4 h$ & 62.5 & $>500$ & $>500$ & $>500$ & $>500$ & $>500$ & $>500$ \\
\hline 10 & $4 i$ & $>500$ & $>500$ & $>500$ & $>500$ & $>500$ & $>500$ & 125 \\
\hline 11 & $4 \mathbf{j}$ & 62.5 & $>500$ & $>500$ & 125 & $>500$ & $>500$ & 125 \\
\hline 12 & $4 k$ & $>500$ & $>500$ & $>500$ & $>500$ & $>500$ & $>500$ & $>500$ \\
\hline 13 & 41 & 62.5 & $>500$ & $>500$ & $>500$ & $>500$ & $>500$ & $>500$ \\
\hline 14 & $4 m$ & $>500$ & $>500$ & $>500$ & $>500$ & $>500$ & $>500$ & 125 \\
\hline 15 & $4 n$ & 62.5 & $>500$ & $>500$ & $>500$ & $>500$ & $>500$ & $>500$ \\
\hline 16 & 40 & 500 & $>500$ & $>500$ & $>500$ & $>500$ & $>500$ & 500 \\
\hline $\begin{array}{c}\text { Ceftriax } \\
\text { one }\end{array}$ & CTX & 0.14 & 2.3 & 18 & 1.17 & 2.4 & 0.58 & 0.29 \\
\hline $\begin{array}{l}\text { Amoxcill } \\
\text { in }\end{array}$ & AMX & 1.56 & 12.5 & 12.5 & - & - & - & 3.1 \\
\hline $\begin{array}{l}\text { Dimethyl } \\
\text { sulfoxide }\end{array}$ & $\begin{array}{c}\text { DMS } \\
\text { O }\end{array}$ & $>500$ & $>500$ & $>500$ & $>500$ & $>500$ & $>500$ & $>500$ \\
\hline
\end{tabular}


Table 2. Average Inhibition of the adherence (\%) of S. aureus ATCC 29213 to HCT 116 ATCC $®$ CCL-247TMhuman colorectal carcinoma cells>

\begin{tabular}{|c|c|c|c|c|c|}
\hline \multirow{2}{*}{ No. } & \multirow{2}{*}{ Code } & \multicolumn{4}{|c|}{ Concentration $(\mu \mathrm{M})$} \\
\hline & & 6.25 & 12.5 & 25 & 50 \\
\hline 1 & 3 & -117.39 & -30.84 & 12.41 & -66.10 \\
\hline 2 & $4 a$ & -30.43 & $77.57 * *$ & $59.12 *$ & $88.14 * *$ \\
\hline 3 & $4 b$ & $69.57 *$ & $86.92 * *$ & $95.62 * *$ & $100.00 * *$ \\
\hline 4 & $4 \mathrm{c}$ & $91.30 * *$ & $98.13 * *$ & $98.54 * *$ & $99.15 * *$ \\
\hline 5 & $4 d$ & -52.17 & $51.40 *$ & $82.48 * *$ & $64.41 *$ \\
\hline 6 & $4 \mathrm{e}$ & 0.00 & $68.22 *$ & $86.13 * *$ & 40.68 \\
\hline 7 & $4 \mathrm{f}$ & 13.04 & 42.06 & 8.03 & 32.20 \\
\hline 8 & $4 g$ & -34.78 & $58.88^{*}$ & $76.64 * *$ & $55.93 *$ \\
\hline 9 & $4 h$ & -134.78 & -15.89 & $88.32 * *$ & 10.17 \\
\hline 10 & $4 \mathrm{i}$ & -52.17 & $64.49 * *$ & $83.94 * *$ & 47.46 \\
\hline 11 & $4 j$ & -213.04 & $51.40 *$ & 27.01 & 5.08 \\
\hline 12 & $4 \mathrm{k}$ & 26.09 & 49.53 & $76.64 * *$ & 44.07 \\
\hline 13 & 41 & -121.74 & -49.53 & 0.73 & -54.24 \\
\hline 14 & $4 \mathrm{~m}$ & 39.13 & $55.14 *$ & 18.25 & 27.12 \\
\hline 15 & $4 n$ & $80.43 * *$ & $66.36^{*}$ & $54.74 *$ & 37.29 \\
\hline 16 & 40 & -78.26 & 4.67 & 19.71 & -22.03 \\
\hline
\end{tabular}

* Significantly different from zero $(p<0.05)$ using one-sample $t$-test; $* *$ highly significantly different from zero $(p<0.01)$ using one-sample $t$-test.

\section{Experimental}

\section{General}

Melting points were determined in open capillary tubes using Electrothermal apparatus (Stuart, UK) and are uncorrected IR spectra were recorded using the potassium bromide method on Perkin-Elmer 1650 spectrophotometer and expressed in wave number (vmax) $\mathrm{cm}^{-1} .{ }^{1} \mathrm{H}-\mathrm{NMR}$ and ${ }^{13} \mathrm{C}$-NMR spectra were measured in deuterated chloroform $\left(\mathrm{CDCl}_{3}\right)$ or deuterated dimethyl sulphoxide (DMSO- $\left.d_{6}\right)$ on Avance III 600 $\mathrm{MHz}$ spectrometer (Bruker, Germany) and chemical shifts were ex-pressed as ppm 
against TMS as internal reference (King Fahd Center for Medical Research, King Abdulaziz University, Jeddah, Saudi Arabia). LCMS were run on Agilent 6130 Series, single quad. HPLC separation was run on 1200 SERIES HPLC using Chemstation Software. Purities were assessed by HPLC and were confirmed to be $>95 \%$ for all final compounds. Reaction progress and compound purity were monitored by Thin Layer Chromatography (TLC) using silica gel matrix, L $\times W 5 \mathrm{~cm} \times 20 \mathrm{~cm}$, fluorescent indicator (Sigma-Aldrich ${ }^{\circledR}$ TLC Plates, USA) and dichloromethane: petroleum ether (4:1) or dichloromethane as eluent systems. The spots were visualized using an ultraviolet lamp (Vilber Lourmet, Marine La Vallee, France) at $\lambda=254$ and $266 \mathrm{~nm}$. Column chromatography was performed on silica gel 60 (particle size $0.06 \mathrm{~mm}-0.20$ $\mathrm{mm})$.

The antibacterial activity of the synthesized compounds was tested against standard strains of seven bacteria. They were obtained from the microbiology laboratory, King Abdulaziz University Hospital, Jeddah, KSA. These strains included Gram-negative bacteria: Escherichia coli ATCC 35218 Bacillus subtilis ATCC 6633, Klebsiella pneumonia ATCC 700603, Pseudomonas aeruginosa ATCC 27853 Staphylococcus epidermidis ATCC 12228 and Gram-positive bacteria: Staphylococcus Aureus ATCC 29213 and P. mirabilis ATCC 14153.

\section{Chemical Synthesis}

Preparation of (Z)-2-(4-fluorophenyl)-4-((E)-3-phenylallylidene)oxazol-5(4H)-one (3):

A mixture of 4-flourobenzoylglycine (1) (1.97 g, $0.01 \mathrm{~mol})$, cinnamaldehyde (2) $(1.98 \mathrm{~g}, 1.88 \mathrm{ml}, 0.015 \mathrm{~mol})$, anhydrous sodium acetate $(0.6 \mathrm{~g}, 0.07 \mathrm{~mol})$ and acetic anhydride $(5 \mathrm{ml})$ was warmed on a boiling water bath with occasional stirring for 20 minutes. The resulting solution was refluxed for 1 hour, cooled and left in a refrigerator for an overnight. The produced yellow solid mass was filtered, washed with cold water then washed with petroleum ether, dried and crystalized from ethanol. Yield $2.5 \mathrm{~g}$ (85\%). M.p. $151-153{ }^{\circ} \mathrm{C}$.

General Procedure for the Preparation of $(Z)-3$-substituted-2-(4-fluorophenyl)-5((E)-3-phenylallylidene)-3,5-dihydro-4H-imidazol-4-one (4)

A mixture of (Z)-2-(4-fluorophenyl)-4-((E)-3-phenylallylidene)oxazol-5(4H)one (3) $(2.93 \mathrm{~g}, 0.01 \mathrm{~mol})$, the appropriate amine $(0.01 \mathrm{~mol})$ and pyridine $(10 \mathrm{ml})$ was taken in a 25 ml-tube of Thermal Integrity Reaction Station and the temperature was kept at $100{ }^{\circ} \mathrm{C}$ for 18 hours. The reaction mixture was poured onto crushed ice and neutralized with drops of conc. $\mathrm{HCl}$. The solid separated out was filtered, washed with water, left to air-dry, then purified by flash column chromatography (silica gel, mixtures of petroleum ether/dichloromethane, 1:4, v/v) to afford the desired pure compounds (4a40).

\section{(Z)-2-(4-Fluorophenyl)-3-phenyl-5-((E)-3-phenylallylidene)-3,5-dihydro-4H- imidazol-4-one (4a):}

The yellow solid crystals were obtained in $68 \%$ yield; m.p. $168{ }^{\circ} \mathrm{C}$. ${ }^{1} \mathrm{H}$ NMR $\left(600 \mathrm{MHz}, \mathrm{CDCl}_{3}\right) \square 7.85(\mathrm{dd}, J=11.86,15.62 \mathrm{~Hz}, 1 \mathrm{H}), 7.65(\mathrm{~d}, J=7.53 \mathrm{~Hz}, 2 \mathrm{H})$, $7.57(\mathrm{dd}, J=5.27,8.66 \mathrm{~Hz}, 2 \mathrm{H}), 7.44-7.50(\mathrm{~m}, 2 \mathrm{H}), 7.40-7.44(\mathrm{~m}, 3 \mathrm{H}), 7.38(\mathrm{~d}, J=$ $7.15 \mathrm{~Hz}, 1 \mathrm{H}), 7.27(\mathrm{~d}, J=11.67 \mathrm{~Hz}, 1 \mathrm{H}), 7.14-7.21(\mathrm{~m}, 3 \mathrm{H}), 7.04(\mathrm{t}, J=8.66 \mathrm{~Hz}, 2 \mathrm{H})$; ${ }^{13} \mathrm{C} \mathrm{NMR}\left(151 \mathrm{MHz}, \mathrm{CDCl}_{3}\right) \square 131.7,131.6,129.8,129.8,129.1,128.7,128.1,127.6$, 
127.5, 123.9, 116.0, 115.9, 77.5, 77.2, 77.0; IR (FT-IR, $\left.\mathrm{cm}^{-1}\right): 3052.9,1736.9,1710.5$, 1602.4, 1489.0, 1365.0, 1296.5; LC-MS (ESI), RT = $7.5 \mathrm{~min}, \mathrm{~m} / z 369.0[\mathrm{M}+\mathrm{H}]^{+}$

\section{(Z)-3-Benzyl-2-(4-fluorophenyl)-5-((E)-3-phenylallylidene)-3,5-dihydro-4H- imidazol-4-one (4b):}

The yellowish solid crystals were obtained in $73 \%$ yield; m.p. $155{ }^{\circ} \mathrm{C} .{ }^{1} \mathrm{H}$ NMR $\left(400 \mathrm{MHz}, \mathrm{CDCl}_{3}\right) \square 7.79(\mathrm{dd}, J=11.80,15.56 \mathrm{~Hz}, 1 \mathrm{H}), 7.60-7.69(\mathrm{~m}, 4 \mathrm{H}), 7.31$ $7.46(\mathrm{~m}, 6 \mathrm{H}), 7.26(\mathrm{~d}, J=11.80 \mathrm{~Hz}, 1 \mathrm{H}), 7.11-7.20(\mathrm{~m}, 5 \mathrm{H}), 4.95(\mathrm{~s}, 2 \mathrm{H}) ;{ }^{13} \mathrm{C} \mathrm{NMR}$ $\left(151 \mathrm{MHz}, \mathrm{CDCl}_{3}\right) \square 136.5,131.1,129.9,129.3,129.2,129.1,128.3,128.1,128.0$, $126.9,126.8,123.8,116.4,116.3,77.5,77.2,77.0,45.5,29.9$; IR (FT-IR, cm ${ }^{-1}$ ): 3043.3, 1709.8, 1630.4, 1598.0, 1495.0, 1362.5, 1238.9; LC-MS (ESI), RT = $11.0 \mathrm{~min}$, $m / z, 383[\mathrm{M}+\mathrm{H}]^{+}$

\section{(Z)-2-(4-Fluorophenyl)-5-((E)-3-phenylallylidene)-3-(pyridin-3-yl)-3,5-dihydro- $4 H$-imidazol-4-one (4c):}

The yellow solid crystals were obtained in $51 \%$ yield; m.p. $175{ }^{\circ} \mathrm{C} .{ }^{1} \mathrm{H}$ NMR $\left(600 \mathrm{MHz}, \mathrm{CDCl}_{3}\right) \square 8.66(\mathrm{~d}, J=4.14 \mathrm{~Hz}, 1 \mathrm{H}), 8.43(\mathrm{~s}, 1 \mathrm{H}), 7.84(\mathrm{dd}, J=11.67,15.43$ $\mathrm{Hz}, 1 \mathrm{H}), 7.66(\mathrm{~d}, J=7.53 \mathrm{~Hz}, 2 \mathrm{H}), 7.58-7.63(\mathrm{~m}, 1 \mathrm{H}), 7.55(\mathrm{dd}, J=5.27,8.28 \mathrm{~Hz}$, $2 \mathrm{H}), 7.35-7.48(\mathrm{~m}, 5 \mathrm{H}), 7.20(\mathrm{~d}, J=15.43 \mathrm{~Hz}, 1 \mathrm{H}), 7.08(\mathrm{t}, J=8.47 \mathrm{~Hz}, 2 \mathrm{H}) ;{ }^{13} \mathrm{C}$ NMR $\left(151 \mathrm{MHz}, \mathrm{CDCl}_{3}\right) \square 137.8,134.3,131.9,131.3,131.3,131.1,129.9,128.9$, 128.0, 128.0, 123.4, 116.2, 116.0, 77.2, 77.0, 76.8 ; IR (FT-IR, $\mathrm{cm}^{-1}$ ): 3071.4, 1279.6, 1716.9, 1600.4, 1494.1, 1433.3, 1365.0, 1296.6; LC-MS (ESI), RT = 5.1 $\mathrm{min}, \mathrm{m} / z .370$ $[\mathrm{M}+\mathrm{H}]^{+}$

\section{(Z)-2-(4-Fluorophenyl)-5-((E)-3-phenylallylidene)-3,5-dihydro-4H-imidazol-4-one} (4d):

The yellowish solid crystals were obtained in $48 \%$ yield; m.p. $292-93{ }^{\circ} \mathrm{C} . ;{ }^{1} \mathrm{H}$ NMR (850 MHz, DMSO-d $\left.)_{6}\right) \square 12.05(\mathrm{~s}, 1 \mathrm{H}), 8.17$ - $8.22(\mathrm{~m}, 2 \mathrm{H}), 8.08-8.14(\mathrm{~m}, 1$ H), 7.62 - $7.70(\mathrm{~m}, 2 \mathrm{H}), 7.58$ (d, J=7.79 Hz, $1 \mathrm{H}), 7.42$ - $7.48(\mathrm{~m}, 4 \mathrm{H}), 7.35-7.41$ (m, 1 H), $7.30(\mathrm{~d}, J=15.57 \mathrm{~Hz}, 1 \mathrm{H}), 6.96(\mathrm{dd}, J=11.42,1.04 \mathrm{~Hz}, 1 \mathrm{H}) ;{ }^{13} \mathrm{C}$ NMR $(214 \mathrm{MHz}$, DMSO-d $\left.{ }_{6}\right) \square 171.4,158.4,141.9,141.7,130.3,130.3,130.0,129.9,129.8,129.6$, 129.5, 127.9, 127.7, 127.3, 125.2, 124.0, 116.7, 116.6; IR (FT-IR, $\left.\mathrm{cm}^{-1}\right)$ : 3132.2, 3063.8, 1691.6, 1630.8, 1504.2, 1438.4, 1230.8, 1159.94; LC-MS (ESI), RT = 4.6 min, $m / z 293[\mathrm{M}+\mathrm{H}]^{+}$

\section{(Z)-2-(4-Fluorophenyl)-3-methyl-5-((E)-3-phenylallylidene)-3,5-dihydro-4H- imidazol-4-one (4e):}

The yellowish solid crystals were obtained in $66 \%$ yield; m.p. $128{ }^{\circ} \mathrm{C} .{ }^{1} \mathrm{H}$ NMR $\left(400 \mathrm{MHz}, \mathrm{CDCl}_{3}\right) \square 7.69-7.91(\mathrm{~m}, 2 \mathrm{H}), 7.55-7.69(\mathrm{~m}, 1 \mathrm{H}), 7.33-7.44(\mathrm{~m}, 2 \mathrm{H}), 7.14$ - $7.32(\mathrm{~m}, 4 \mathrm{H}), 3.31-3.42(\mathrm{~m}, 2 \mathrm{H}), 1.61(\mathrm{~s}, 3 \mathrm{H}) ;{ }^{13} \mathrm{C}$ NMR $(214 \mathrm{MHz}$, DMSO-d 6$) \square$ 165.62, 164.36, 160.40, 160.3, 159.21, 159.13, 155.12, 154.44, 139.09, 138.14, 134.68, 131.55, 126.07, 120.75, 118.80, 24.37; IR (FT-IR, $\mathrm{cm}^{-1}$ ): 3038.56, 1709.3, 1628.3, 1597.9, 1504.2, 1362.4, 1230.8; LC-MS (ESI), RT = $5.4 \mathrm{~min}, \mathrm{~m} / z 306.9[\mathrm{M}+\mathrm{H}]^{+}$

(Z)-2-(4-Fluorophenyl)-3-(4-methylpiperazin-1-yl)-5-((E)-3-phenylallylidene)-3,5dihydro-4H-imidazol-4-one (4f): 
The yellowish solid crystals were obtained in $57 \%$ yield; m.p. $231-33{ }^{\circ} \mathrm{C} .{ }^{1} \mathrm{H}$ $\operatorname{NMR}\left(600 \mathrm{MHz}, \mathrm{CDCl}_{3}\right) \square 8.30(\mathrm{dd}, J=5.65,9.03 \mathrm{~Hz}, 2 \mathrm{H}), 7.76(\mathrm{dd}, J=11.67,15.43$ $\mathrm{Hz}, 1 \mathrm{H}), 7.61-7.65(\mathrm{~m}, 2 \mathrm{H}), 7.34-7.45(\mathrm{~m}, 2 \mathrm{H}), 7.29$ (s, 3H), $7.18-7.23(\mathrm{~m}, 2 \mathrm{H})$, 7.08 - 7.13 (m, 2H), 4.14 (br. s., 2H), 2.39 (br. s., 4H), 1.65 (br. s., 3H); IR (FT-IR, $\left.\mathrm{cm}^{-1}\right): 2928.5,2866.7,2787.2,1704.0,1624.5,1603.9,1495.0,1453.8,1283.0,1230.0$; LC-MS (ESI), RT = $4.9 \mathrm{~min}, \mathrm{~m} / z 391.0[\mathrm{M}+\mathrm{H}]^{+}$

(Z)-2-(4-Fluorophenyl)-5-((E)-3-phenylallylidene)-3-propyl-3,5-dihydro-4Himidazol-4-one (4g):

The yellowish solid crystals were obtained in $65 \%$ yield; m.p. $119{ }^{\circ} \mathrm{C} .{ }^{1} \mathrm{H}$ NMR $\left(400 \mathrm{MHz}, \mathrm{CDCl}_{3}\right) \square 7.67-7.86(\mathrm{~m}, 3 \mathrm{H}), 7.62(\mathrm{~d}, J=7.28 \mathrm{~Hz}, 2 \mathrm{H}), 7.33-7.43(\mathrm{~m}$, $3 \mathrm{H}), 7.09-7.31(\mathrm{~m}, 4 \mathrm{H}), 3.66-3.81(\mathrm{~m}, 2 \mathrm{H}), 1.52-1.78(\mathrm{~m}, 3 \mathrm{H}), 0.88(\mathrm{t}, J=7.40 \mathrm{~Hz}$, $3 \mathrm{H}) ;{ }^{13} \mathrm{C}$ NMR $\left(214 \mathrm{MHz}, \mathrm{CDCl}_{3}\right) \square 163.9,160.1,136.3,130.6,129.6,128.9,128.7$, 128.4, 127.9, 124.2, 124.1, 123.6, 116.4, 116.3, 116.0, 43.4, 43.3, 22.4, 22.0, 11.2, 11.1; IR (FT-IR, $\mathrm{cm}^{-1}$ ): 2971.5, 2932.0, 2869.0, 1701.7, 1628.1, 1628.1, 1599.2, 1499.3, 1281.1, 1228.5; LC-MS (ESI), RT = $8.1 \mathrm{~min}, \mathrm{~m} / \mathrm{z} 335.0[\mathrm{M}+\mathrm{H}]^{+}$

\section{3-((Z)-2-(4-Fluorophenyl)-5-oxo-4-((E)-3-phenylallylidene)-4,5-dihydro-1H- imidazol-1-yl)benzonitrile (4h):}

The yellowish solid crystals were obtained in $72 \%$ yield; m.p. $203-05{ }^{\circ} \mathrm{C} .{ }^{1} \mathrm{H}$ NMR $\left(600 \mathrm{MHz}, \mathrm{CDCl}_{3}\right) \square 7.84(\mathrm{dd}, J=11.67,15.81 \mathrm{~Hz}, 1 \mathrm{H}), 7.61-7.73(\mathrm{~m}, 3 \mathrm{H})$, 7.51 - $7.60(\mathrm{~m}, 3 \mathrm{H}), 7.36$ - $7.50(\mathrm{~m}, 4 \mathrm{H}), 7.27$ - $7.32(\mathrm{~m}, 2 \mathrm{H}), 7.13$ - $7.25(\mathrm{~m}, 1 \mathrm{H}), 6.99$ 7.13 (m, 2H); ${ }^{13} \mathrm{C}$ NMR $\left(151 \mathrm{MHz}, \mathrm{CDCl}_{3}\right) \square 136.3,132.5,131.7,131.5,131.5,130.6$, 130.1, 129.2, 128.3, 128.2, 123.6, 116.5, 116.3, 77.5, 77.2, 77.0 ; IR (FT-IR, cm ${ }^{-1}$ ): 3066.1, 2235.3, 1707.9, 1631.4, 1496.9, 1481.1, 1370.3, 1238.4, 1151.4; LC-MS (ESI), $\mathrm{RT}=7.1 \mathrm{~min}, m / z, 394.0[\mathrm{M}+\mathrm{H}]^{+}$

\section{(Z)-2-(4-Fluorophenyl)-3-(4-hydroxyphenyl)-5-((E)-3-phenylallylidene)-3,5- dihydro-4H-imidazol-4-one (4i):}

The yellowish solid crystals were obtained in $57 \%$ yield; m.p. $92-934{ }^{\circ} \mathrm{C} .{ }^{1} \mathrm{H}$ NMR $\left(600 \mathrm{MHz}, \mathrm{CDCl}_{3}\right) \square 7.85(\mathrm{dd}, J=11.67,15.81 \mathrm{~Hz}, 1 \mathrm{H}), 7.62-7.66(\mathrm{~m}, 1 \mathrm{H})$, 7.58 - $7.62(\mathrm{~m}, 1 \mathrm{H}), 7.37$ - $7.44(\mathrm{~m}, 3 \mathrm{H}), 7.26-7.30(\mathrm{~m}, 2 \mathrm{H}), 7.16-7.21(\mathrm{~m}, 1 \mathrm{H}), 6.96-$ 7.04 (m, 4H), 6.77 - 6.83 (m, 2H) ${ }^{13} \mathrm{C}$ NMR $\left(151 \mathrm{MHz}, \mathrm{CDCl}_{3}\right) \square$ 156.8, 136.4, 133.0, $133.0,131.9,131.7,131.7,130.1,129.2,129.1,128.9,128.9,128.3,128.2,123.8$, 117.0, 117.0, 116.1, 116.0, 115.9, 115.8, 77.5, 77.2, 77.0; IR (FT-IR, $\left.\mathrm{cm}^{-1}\right)$ : 3029.2, $1707.9,1692.1,1626.1,1594.5,1510.1,1370.3,1272.7,1222.6,1156.7$; $\quad$ LC-MS (ESI), RT $=4.9 \mathrm{~min}, \mathrm{~m} / \mathrm{z} 385.0[\mathrm{M}+\mathrm{H}]^{+}$

\section{(Z)-2,3-bis(4-Fluorophenyl)-5-((E)-3-phenylallylidene)-3,5-dihydro-4H-imidazol-4- one $(\mathbf{4 j})$ :}

The yellowish solid crystals were obtained in $57 \%$ yield; m.p. $135-37{ }^{\circ} \mathrm{C} . ;{ }^{1} \mathrm{H}$ NMR (850 MHz, DMSO-d 6 ) $\square 7.71-7.75(\mathrm{~m}, 1 \mathrm{H}), 7.69$ (d, $J=7.27 \mathrm{~Hz}, 1 \mathrm{H}), 7.59(\mathrm{~d}, J$ $=7.79 \mathrm{~Hz}, 1 \mathrm{H}), 7.54-7.57(\mathrm{~m}, 1 \mathrm{H}), 7.47-7.50(\mathrm{~m}, 1 \mathrm{H}), 7.45(\mathrm{t}, J=7.53 \mathrm{~Hz}, 2 \mathrm{H}), 7.39$ - $7.43(\mathrm{~m}, 2 \mathrm{H}), 7.31-7.39(\mathrm{~m}, 5 \mathrm{H}), 7.23-7.29(\mathrm{~m}, 2 \mathrm{H})$; IR (FT-IR, $\left.\mathrm{cm}^{-1}\right)$ : 3058.2, 1712.2, 1628.8, 1507.4, 1415.1, 1285.9, 1222.6, 1151.4; LC-MS (ESI), RT = $9.0 \mathrm{~min}$, $\mathrm{m} / \mathrm{z} 386.9[\mathrm{M}+\mathrm{H}]^{+}$ 
(Z)-2-(4-Fluorophenyl)-3-(furan-2-ylmethyl)-5-((E)-3-phenylallylidene)-3,5dihydro-4H-imidazol-4-one (4k):

The yellowish solid crystals were obtained in $48 \%$ yield; m.p. $145{ }^{\circ} \mathrm{C} .{ }^{1} \mathrm{H}$ NMR $\left(400 \mathrm{MHz}, \mathrm{CDCl}_{3}\right) \square 7.62-7.76(\mathrm{~m}, 2 \mathrm{H}), 7.48-7.55(\mathrm{~m}, 1 \mathrm{H}), 7.05-7.32(\mathrm{~m}, 7 \mathrm{H}), 6.12$ - $6.26(\mathrm{~m}, 1 \mathrm{H}), 4.80(\mathrm{~s}, 1 \mathrm{H}), 1.51(\mathrm{~s}, 4 \mathrm{H})$; LC-MS (ESI), RT = $7.3 \mathrm{~min}, \mathrm{~m} / z 373.0[\mathrm{M}+$ $\mathrm{H}]^{+}$

(Z)-2-(4-Fluorophenyl)-3-(2-morpholinoethyl)-5-((E)-3-phenylallylidene)-3,5dihydro-4H-imidazol-4-one (4l):

The yellowish solid crystals were obtained in $44 \%$ yield; m.p. $163{ }^{\circ} \mathrm{C} .{ }^{1} \mathrm{H}$ NMR $\left(400 \mathrm{MHz}, \mathrm{CDCl}_{3}\right) \square 7.82-7.89(\mathrm{~m}, 2 \mathrm{H}), 7.62(\mathrm{~d}, J=7.28 \mathrm{~Hz}, 2 \mathrm{H}), 7.35-7.44(\mathrm{~m}$, 3H), 7.28 (br. s., 1H), $7.22-7.27(\mathrm{~m}, 2 \mathrm{H}), 7.10-7.21(\mathrm{~m}, 2 \mathrm{H}), 3.90(\mathrm{t}, J=6.27 \mathrm{~Hz}$, 2H), $3.54-3.61(\mathrm{~m}, 4 \mathrm{H}), 2.50(\mathrm{t}, J=6.27 \mathrm{~Hz}, 2 \mathrm{H}), 2.31-2.37(\mathrm{~m}, 4 \mathrm{H})$; IR (FT-IR, cm ${ }^{-1}$ ): 3072.7, 2960.9, 2796.0, 1704.0, 1630.4, 1506.7, 1418.4, 1350.7, 1224.2, 1150.6; LCMS (ESI), RT $=4.9 \mathrm{~min}, m / z, 406.0[\mathrm{M}+\mathrm{H}]^{+}$

(Z)-2-(4-Fluorophenyl)-3-(4-methoxyphenyl)-5-((E)-3-phenylallylidene)-3,5dihydro-4H-imidazol-4-one $(4 \mathrm{~m})$ :

The yellowish solid crystals were obtained in $48 \%$ yield; m.p. $198-200{ }^{\circ} \mathrm{C} .{ }^{1} \mathrm{H}$ NMR $\left(600 \mathrm{MHz}, \mathrm{CDCl}_{3}\right) \square 7.84(\mathrm{dd}, J=11.67,15.81 \mathrm{~Hz}, 1 \mathrm{H}), 7.65(\mathrm{~d}, J=7.15 \mathrm{~Hz}$, $1 \mathrm{H}), 7.58-7.63(\mathrm{~m}, 2 \mathrm{H}), 7.55(\mathrm{dd}, J=5.27,8.66 \mathrm{~Hz}, 1 \mathrm{H}), 7.40-7.45(\mathrm{~m}, 1 \mathrm{H}), 7.34-$ $7.40(\mathrm{~m}, 1 \mathrm{H}), 7.27-7.28(\mathrm{~m}, 1 \mathrm{H}), 7.23-7.27(\mathrm{~m}, 1 \mathrm{H}), 7.08-7.17(\mathrm{~m}, 3 \mathrm{H}), 7.01-7.07$ (m, 2H), $6.95-7.00$ (m, 2H), 3.85 (s, 3H); IR (FT-IR, $\mathrm{cm}^{-1}$ ): 3010.4, 1709.51, 1638.6, 1547.5, 1441.2, 1415.9, 1357.9, 1215.9; LC-MS (ESI), RT = $8.9 \mathrm{~min}, \mathrm{~m} / \mathrm{z} 399.0[\mathrm{M}+$ $\mathrm{H}]^{+}$

(Z)-3-(4-Chlorophenyl)-2-(4-fluorophenyl)-5-((E)-3-phenylallylidene)-3,5-dihydro4H-imidazol-4-one (4n):

The yellowish solid crystals were obtained in $65 \%$ yield; m.p. $198^{\circ} \mathrm{C} .{ }^{1} \mathrm{H}$ NMR $\left(600 \mathrm{MHz}, \mathrm{CDCl}_{3}\right) \square 7.84(\mathrm{dd}, J=11.67,15.81 \mathrm{~Hz}, 1 \mathrm{H}), 7.65(\mathrm{~d}, J=7.15 \mathrm{~Hz}, 2 \mathrm{H})$, 7.57 (dd, $J=5.46,8.85 \mathrm{~Hz}, 2 \mathrm{H}), 7.37-7.45(\mathrm{~m}, 5 \mathrm{H}), 7.27$ (d, $J=11.67 \mathrm{~Hz}, 1 \mathrm{H}), 7.18$ $(\mathrm{d}, J=15.81 \mathrm{~Hz}, 1 \mathrm{H}), 7.13(\mathrm{~d}, J=8.66 \mathrm{~Hz}, 2 \mathrm{H}), 7.08(\mathrm{t}, J=8.66 \mathrm{~Hz}, 2 \mathrm{H}) ;{ }^{13} \mathrm{C} \mathrm{NMR}$ $\left(214 \mathrm{MHz}, \mathrm{CDCl}_{3}\right) \square 168.8,165.1,163.9,157.4,143.7,136.2,134.3,132.9,131.5$, $131.4,131.3,129.8,128.9,128.5,128.4,128.0,127.9,124.6,124.0,123.5,116.0$, 116.0, 115.9, 115.9; ); IR (FT-IR, $\mathrm{cm}^{-1}$ ): 3063.6, 1729.6, 1719.4, 1636.8, 1595.4, 1494.1, 1413.1, 1365.0, 1238.4; LC-MS (ESI), RT = $11.8 \mathrm{~min}, \mathrm{~m} / z 403.0[\mathrm{M}+\mathrm{H}]^{+}$

(Z)-2-(4-Fluorophenyl)-3-(3-methoxyphenyl)-5-((E)-3-phenylallylidene)-3,5dihydro-4H-imidazol-4-one (4o):

The yellowish solid crystals were obtained in $42 \%$ yield; m.p. $119^{\circ} \mathrm{C} .{ }^{1} \mathrm{H}$ NMR $\left(600 \mathrm{MHz}, \mathrm{CDCl}_{3}\right) \square 7.84(\mathrm{dd}, J=11.67,15.81 \mathrm{~Hz}, 1 \mathrm{H}), 7.57-7.69(\mathrm{~m}, 3 \mathrm{H}), 7.55$ (dd, $J=5.27,8.66 \mathrm{~Hz}, 1 \mathrm{H}), 7.32-7.46(\mathrm{~m}, 4 \mathrm{H}), 7.26(\mathrm{~d}, J=11.29 \mathrm{~Hz}, 1 \mathrm{H}), 7.09-7.19(\mathrm{~m}$, 1H), 7.01 - $7.08(\mathrm{~m}, 2 \mathrm{H}), 6.94-7.00(\mathrm{~m}, 1 \mathrm{H}), 6.71$ - $6.79(\mathrm{~m}, 2 \mathrm{H}), 3.78(\mathrm{~s}, 3 \mathrm{H})$; IR (FT$\left.\mathrm{IR}, \mathrm{cm}^{-1}\right): 2838.5,1929.6,1714.4,1628.0,1595.4,1489.0,1299.1,1225.7,1152.34$; LC-MS (ESI), RT = $8.8 \mathrm{~min}, \mathrm{~m} / z, 299[\mathrm{M}+\mathrm{H}]^{+}$ 


\section{Antibacterial Evaluation}

The antibacterial activities were evaluated regarding the minimum inhibitory concentrations (MICs) by using microbroth dilution assays according to the CLSI guidelines(Wayne, 2006). Using DMSO to dissolve all of the tested compounds, it was also used as negative control with concentrations range from 0.12 to $500 \mu \mathrm{g} / \mathrm{mL}$. Commercial antibiotics amoxicillin, ceftriaxone and nystatin were used as a positive control. The bacterial stock cultures were maintained on Muller-Hinton agar plates. A loopful of overnight bacterial cells from the agar plates was inoculated into $5 \mathrm{~mL}$ normal saline $(85 \% \mathrm{NaCl})$ and turbidity was adjusted to $1-5 \times 10^{6} \mathrm{CFU} / \mathrm{ml}$. Ten $\mu \mathrm{L}$ of standardized bacterial culture was introduced into 96 wells tissue culture plates containing $100 \mu \mathrm{L}$ Muller-Hinton broth/well with various concentrations of the compounds reviewed. The MIC was defined as the lowest possible concentration which could inhibit the growth of the bacterial strains. All of the analysis were performed in triplicate and MIC's values are given in $\mu \mathrm{g} / \mathrm{mL}$.

\section{Adherence assay of $S$. aureus ATCC 29213 to HCT 116 cells}

One hundred microliters of the bacterial suspension (1-5 X $\left.10^{8} \mathrm{CFU} / \mathrm{ml}\right)$ were added to HCT 116 ATCC ${ }^{\circledR}$ CCL-247TM (Human colorectal carcinoma) (Supplied from King Fahd Research center, Jeddah, KSA) in tissue culture plate wells containing 100 $\mu \mathrm{L}$ antibiotic free RPMI 1640 media (Gibco, ThermoFisher Scientific, MA, USA).. Then incubated for 2 hour, the monolayers with attached bacteria were washed three times with phosphate buffer saline (PBS) (Gibco, ThermoFisher Scientific, MA, USA). The total number of the cells (adherent to and uptaken by HCT 116 cells) and the number of the cells uptaken by the HCT 116 cells were determined. Then, by substracting the number of bacterial cells uptaken by the HCT 116 cells from the total number of cells, the number of adherent cells can be determined. The total number of the cell was determined as follows; after addition of the bacterial suspension on HCT 116 and incubation for 2 hours, the monolayers with attached bacteria were washed three times with PBS. Then, lysis of the epithelial cell was carried out by treating with PBS containing $0.025 \%$ trypsin (Sigma-Aldrich, Germany) and 1\% tween 20 (SigmaAldrich, Germany) for $30 \mathrm{~min}$ at $37{ }^{\circ} \mathrm{C}$. Aliquots of the cell lysates were diluted and placed on Trypticase Soy agar to quantify the number of viable cells. The number of uptaken cells was determined as follows; after incubation and washing 3 times with PBS, the monolayers with attached cell were rinsed with tissue culture medium (as described above) containing $300 \mu \mathrm{g} / \mathrm{ml}$ gentamicin for 1 hour to kill all the external bacterial cell. After removal of antibiotic containing medium, the HCT 116 cells were washed and treated with the lysis solution (as described above). Then, the number of cells uptaken can be determined by viable count technique. Assays were carried out in triplicate. The the number of adhered bacteria $\left(\mathrm{CFU} \cdot \mathrm{mL}^{-1}\right)$ relative to the total number of bacteria added initially $\times 100$ was used to calculate the adherence percentage $(\%)$. The percentage of inhibition by compounds was calculated as $[1-(\%$ Adherence sample/\% Adherence control)] $\times 100$.

\section{REFERENCES}

Adams, T. B., Cohen, S. M., Doull, J., Feron, V. J., Goodman, J. I., Marnett, L. J., Munro, I. C., Portoghese, P. S., Smith, R. L. and Waddell, W. J. (2004) 'The FEMA GRAS assessment of cinnamyl derivatives used as flavor ingredients', Food and chemical toxicology, 42(2), pp. 157-185. 
Amalaradjou, M. A. R., Narayanan, A. and Venkitanarayanan, K. (2011) 'Transcinnamaldehyde decreases attachment and invasion of uropathogenic Escherichia coli in urinary tract epithelial cells by modulating virulence gene expression', The Journal of urology, 185(4), pp. 1526-1531.

Anderl, J. N., Franklin, M. J. and Stewart, P. S. (2000) 'Role of antibiotic penetration limitation in Klebsiella pneumoniae biofilm resistance to ampicillin and ciprofloxacin', Antimicrobial agents and chemotherapy, 44(7), pp. 1818-1824.

Brackman, G., Defoirdt, T., Miyamoto, C., Bossier, P., Van Calenbergh, S., Nelis, H. and Coenye, T. (2008) 'Cinnamaldehyde and cinnamaldehyde derivatives reduce virulence in Vibrio spp. by decreasing the DNA-binding activity of the quorum sensing response regulator LuxR', BMC microbiology, 8(1), pp. 149.

Costerton, J. W., Stewart, P. S. and Greenberg, E. P. (1999) 'Bacterial biofilms: a common cause of persistent infections', Science, 284(5418), pp. 1318-22.

Dakin, H. (1929) 'The condensation of aromatic aldehydes with glycine and acetylglycine', The Journal of Biological Chemistry, 82, pp. 439-446.

Dunne, W. M. (2002) 'Bacterial adhesion: seen any good biofilms lately?', Clinical microbiology reviews, 15(2), pp. 155-166.

El-Araby, M., Omar, A., Hassanein, H. H., El-Helby, A. G. and Abdel-Rahman, A. A. (2012) 'Design, synthesis and in vivo anti-inflammatory activities of 2,4-diaryl5-4H-imidazolone derivatives', Molecules, 17(10), pp. 12262-75.

Estela, C. R. L. and Alejandro, P. R. (2012) Biofilms: A Survival and Resistance Mechanism of Microorganisms. INTECH Open Access Publisher.

Foulston, L., Elsholz, A. K., DeFrancesco, A. S. and Losick, R. (2014) 'The extracellular matrix of Staphylococcus aureus biofilms comprises cytoplasmic proteins that associate with the cell surface in response to decreasing $\mathrm{pH}$, MBio, 5(5), pp. e01667-14.

Jensen, P. Ø., Bjarnsholt, T., Phipps, R., Rasmussen, T. B., Calum, H., Christoffersen, L., Moser, C., Williams, P., Pressler, T. and Givskov, M. (2007) 'Rapid necrotic killing of polymorphonuclear leukocytes is caused by quorum-sensing-controlled production of rhamnolipid by Pseudomonas aeruginosa', Microbiology, 153(5), pp. 1329-1338.

Karaman, M., Firıncı, F., Arıkan, A. Z. and Bahar, I. (2013) '[Effects of Imipenem, Tobramycin and Curcumin on Biofilm Formation of Pseudomonas aeruginosa Strains]', Mikrobiyoloji bulteni, 47(1), pp. 192-194.

Khan, S., Singh, P., Ansari, M. and Asthana, A. (2014) 'Isolation of Shigella species and their resistance patterns to a panel of fifteen antibiotics in mid and far western region of Nepal', Asian Pacific Journal of Tropical Disease, 4(1), pp. 30-34.

Kim, S.-M., Lee, H.-W., Choi, Y.-W., Kim, S.-H., Lee, J.-C., Lee, Y.-C., Seol, S.-Y., Cho, D.-T. and Kim, J. (2012) 'Involvement of curli fimbriae in the biofilm formation of Enterobacter cloacae', The Journal of Microbiology, 50(1), pp. 175178. 
Larson, E. L., Gomez-Duarte, C., Lee, L. V., Della-Latta, P., Kain, D. J. and Keswick, B. H. (2003) 'Microbial flora of hands of homemakers', American journal of infection control, 31(2), pp. 72-79.

Lewis, K. (2001) 'Riddle of biofilm resistance', Antimicrobial agents and chemotherapy, 45(4), pp. 999-1007.

Liu, Y.-Q., Liu, Y. and Tay, J.-H. (2004) 'The effects of extracellular polymeric substances on the formation and stability of biogranules', Applied Microbiology and Biotechnology, 65(2), pp. 143-148.

Lokhandwala, S. and Rai, S. 'Synthesis And In Vitro Microbial Evaluation Of Some Novel Imidozol-5-one Derivatives'.

Lyczak, J. B., Cannon, C. L. and Pier, G. B. (2002) 'Lung infections associated with cystic fibrosis', Clinical microbiology reviews, 15(2), pp. 194-222.

Naves, P., Del Prado, G., Huelves, L., Rodriguez-Cerrato, V., Ruiz, V., Ponte, M. and Soriano, F. (2010) 'Effects of human serum albumin, ibuprofen and N-acetyl-Lcysteine against biofilm formation by pathogenic Escherichia coli strains', Journal of Hospital Infection, 76(2), pp. 165-170.

Nickel, J. C. and Costerton, J. W. (1993) 'Bacterial localization in antibiotic- refractory chronic bacterial prostatitis', The Prostate, 23(2), pp. 107-114.

Novick, R. P. and Geisinger, E. (2008) 'Quorum sensing in staphylococci', Annual review of genetics, 42 , pp. 541-564.

Otto, M., Süßmuth, R., Jung, G. and Götz, F. (1998) 'Structure of the pheromone peptide of the Staphylococcus epidermidis agr system', FEBS letters, 424(1), pp. 89-94.

Palaska, I., Papathanasiou, E. and Theoharides, T. C. (2013) 'Use of polyphenols in periodontal inflammation', European journal of pharmacology, 720(1), pp. 77-83.

Plotkowski, M.-C., Saliba, A. M., Pereira, S., Cervante, M. and Bajolet-Laudinat, O. (1994) 'Pseudomonas aeruginosa selective adherence to and entry into human endothelial cells', Infection and immunity, 62(12), pp. 5456-5463.

Sekhar, S., Kumar, R. and Chakraborti, A. (2009) 'Role of biofilm formation in the persistent colonization of Haemophilus influenzae in children from northern India', Journal of medical microbiology, 58(11), pp. 1428-1432.

Smith-Palmer, A., Stewart, J. and Fyfe, L. (2004) 'Influence of subinhibitory concentrations of plant essential oils on the production of enterotoxins A and B and $\alpha$-toxin by Staphylococcus aureus', Journal of medical microbiology, 53(10), pp. 1023-1027.

Stewart, P. S. and Franklin, M. J. (2008) 'Physiological heterogeneity in biofilms', Nature Reviews Microbiology, 6(3), pp. 199-210.

Vadyvaloo, V. and Otto, M. (2005) 'Molecular genetics of Staphylococcus epidermidis biofilms on indwelling medical devices', The International journal of artificial organs, 28(11), pp. 1069-1078. 
Wayne, P. 2006. Clinical and Laboratory Standarts Institute: Methods for Dilution Antimicrobial Susceptibility Tests for Bacteria that grow Aerobically. Approved Standart M7-A7, CLSI, USA.

\section{تصميم وتثييا وتقييم المضادات المحتملة للبكتيريا والالتصاق مختتبريا لبعض الإميدازولون المشتقة من السينمالاد هيا}

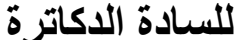

عبد الستار منصور عبيد عمرا، ب* ومحمود عبدالخالق الفقى جالته مـــــ

'قسم الكيمياء الصيدلية، كلية الصيدلة، جامعة الملك عبدالعزيز، السليمانية، جدة 1019 1 ، المملكة العربية السعودية.

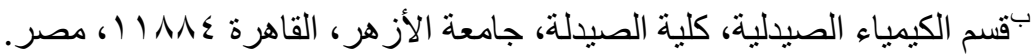

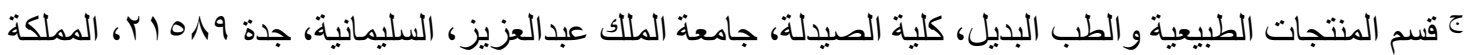

$$
\text { العربية السعودية. }
$$

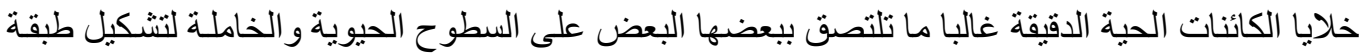

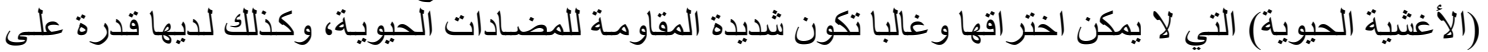

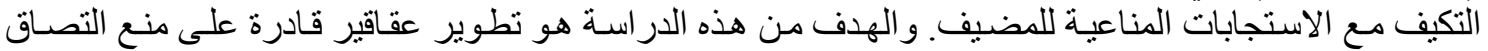

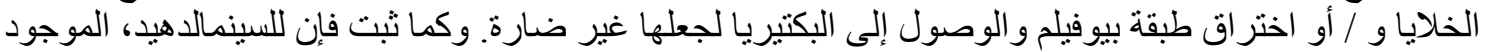

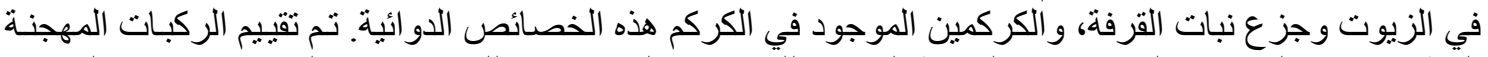

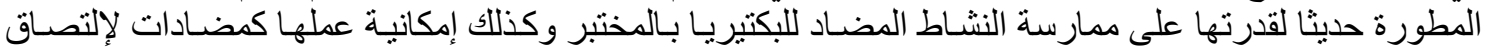

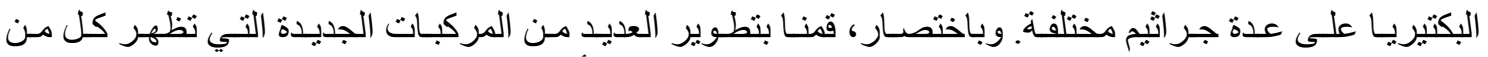

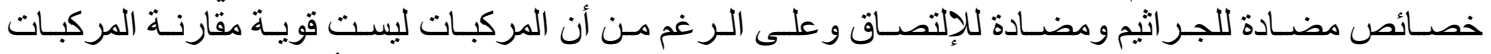
القياسيةولكنها تعتبر بمثابة ركيزة مهمة لمزيد من التعديل الهيكلي للحصول على على مشتقات أكثر فعالية. 